Cite this: Phys. Chem. Chem. Phys., 2011, 13, 13341-13348

\title{
Tethered bilayer lipid micromembranes for single-channel recording: the role of adsorbed and partially fused lipid vesicles
}

\author{
Lucia Becucci, ${ }^{a}{ }^{a}$ Massimo D’Amico, ${ }^{b}$ Serena Cinotti, ${ }^{a}$ Salvatore Daniele ${ }^{c}$ and \\ Rolando Guidelli ${ }^{a}$
}

Received 8th March 2011, Accepted 2nd June 2011

DOI: $10.1039 / \mathrm{c1cp20667b}$

A mercury-supported bilayer lipid micromembrane was prepared by anchoring a thiolipid monolayer to a mercury cap electrodeposited on a platinum microdisc about $20 \mu \mathrm{m}$ in diameter; a lipid monolayer was then self-assembled on top of the thiolipid monolayer either by vesicle fusion or by spilling a few drops of a lipid solution in chloroform on the cap and allowing the solvent to evaporate. Single-channel recording following incorporation of the alamethicin channel-forming peptide exhibits quite different features, depending on the procedure followed to form the distal lipid monolayer. The "spilling" procedure, which avoids the formation of adsorbed or partially fused vesicles, yields very sharp single-channel currents lasting only one or two milliseconds. These are ascribed to ionic flux into the hydrophilic spacer moiety of the thiolipid. Conversely, the vesicle-fusion procedure yields much longer single-channel openings analogous to those obtained with conventional bilayer lipid membranes, albeit smaller.

This difference in behavior is explained by ascribing the latter single-channel currents to ionic flux into vesicles adsorbed and/or partially fused onto the tethered lipid bilayer, via capacitive coupling.

\section{Introduction}

The preparation of rugged lipid bilayers capable of incorporating bulky membrane proteins has been the subject of extensive research. The possibility of using these "biomimetic membranes" for the investigation of the function of membrane proteins is of paramount importance. Ion pumps and, more often, ion channels have been investigated in lipid bilayers tethered to a metal support via a hydrophilic "spacer" (tethered bilayer lipid membranes: tBLMs). ${ }^{1}$ Thanks to their particular robustness, tBLMs have also potential for biosensor applications. They are obtained by anchoring to the metal surface a "thiolipid", which consists of a polyethyleneoxy or oligopeptide hydrophilic chain (the spacer) terminated at one end with a sulfhydryl or disulfide group, for anchoring to the metal surface, and at the other end with two hydrocarbon tails. A lipid monolayer is then self-assembled on top of the thiolipid monolayer, with the polar heads of the lipid turned towards

${ }^{a}$ Department of Chemistry, Florence University, Via della Lastruccia 3, 50019 Sesto Fiorentino, Firenze, Italy.

E-mail:lucia.becucci@unifi.it, serena.cinotti@yahoo.it, guidelli@unifi.it; Fax: (+39) 055-457-3385

Tel: $(+39)$ 055-457-3095

${ }^{b}$ Department of Experimental Pathology and Oncology,

Florence University, Viale G.B. Morgagni 50, 50134 Firenze, Italy. E-mail: massimo.damico@unifi.it

${ }^{c}$ Department of Physical Chemistry, Venice University, Calle Larga

S. Marta 2137,30123 Venezia, Italy.E-mail: sig@unive.it the aqueous solution. This procedure gives rise to a lipid bilayer interposed between the spacer and the aqueous solution. A convenient thiolipid, called DPTL, consists of a hydrophilic tetraethyleneoxy (TEO) chain covalently linked to a lipoic acid residue for anchoring to the metal at one end, and to two phytanyl chains at the other end. ${ }^{2}$ Fig. 1 shows the structural formula of a DPTL molecule tail-to-tail with a dioleoylphosphatidylcholine (DOPC) molecule, namely the assembly that constitutes the building block of the tBLM. Au-supported oligopeptide-based tBLMs have been reported to incorporate a few bulky proton pumps, such as $\mathrm{F}_{0} \mathrm{~F}_{1}$ ATPase ${ }^{3,4}$ and cytochrome $c$ oxidase $(\mathrm{COX}){ }^{5}$ In all these cases, the lipid monolayer on top of the thiolipid monolayer was formed from a suspension of vesicles, via vesicle splitting and spreading on the thiolipid monolayer; the proton pump was then incorporated in the tBLM from its aqueous solution in detergent. Alternatively, the lipid film was formed by fusion of proteoliposomes containing the integral protein under study. The current following activation of the proton pump was assumed to be due to proton flux to or from the hydrophilic spacer through the membrane protein spanning the lipid bilayer.

However, thiolipid-based tBLMs, when anchored to solid supports such as gold, do not exhibit the fluidity and lateral mobility required for the incorporation of bulky membrane proteins. The thiolipid molecules are rigidly bound to the metal surface atoms and cannot make room for these proteins. 


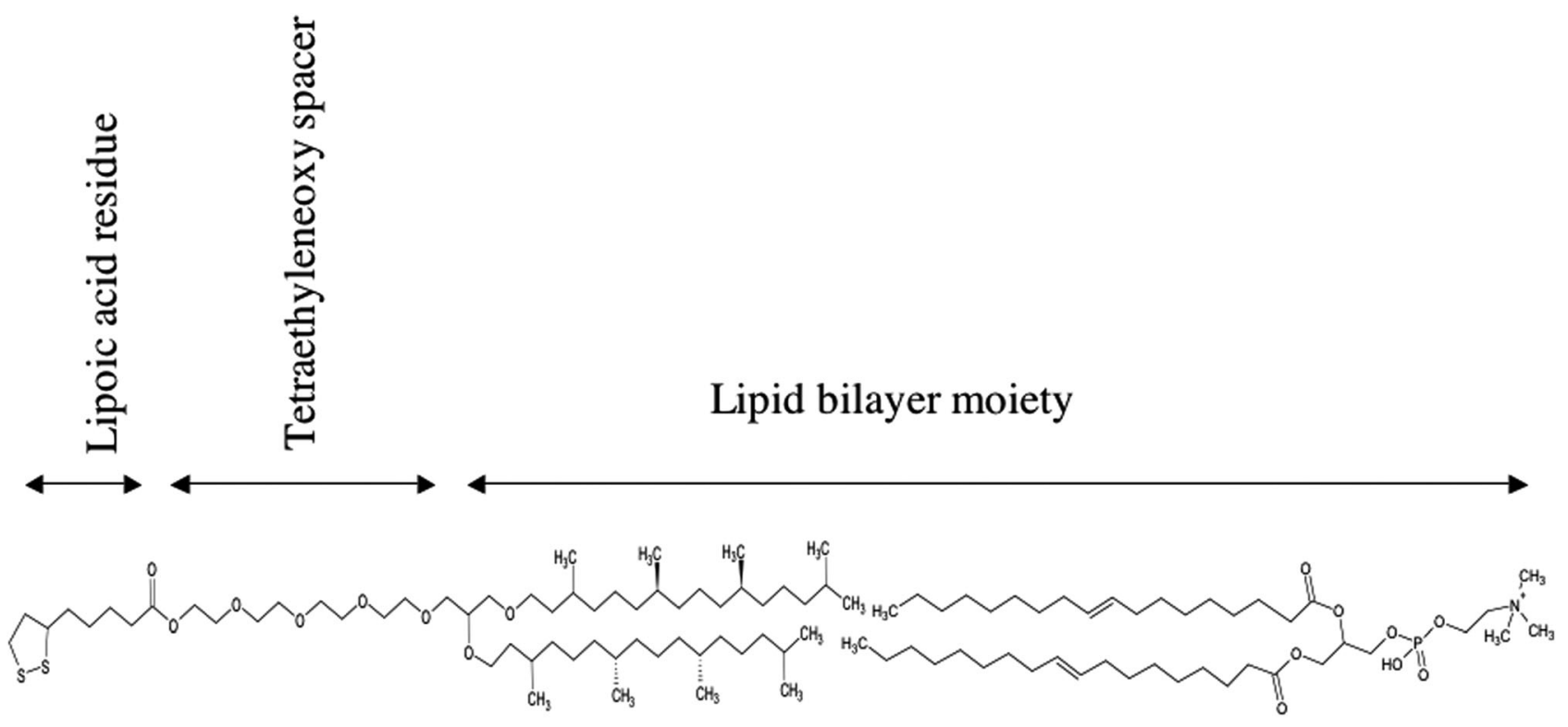

Fig. 1 Structural formula of a DPTL molecule tail-to-tail with a DOPC molecule.

On the other hand, membrane proteins should span the whole tBLM, including the thiolipid monolayer, in order to translocate ions into the hydrophilic spacer and to give rise to a resulting capacitive or faradaic current. Moreover, the hydration of the hydrophilic moiety of thiolipids anchored to gold is low, ${ }^{6,7}$ while the incorporation of proteins with extramembrane domains requires a significant hydration of the spacer. In principle, the lipid molecules on top of the thiolipid monolayer might be free to move laterally. In practice, however, their lateral mobility is hindered by the presence of adsorbed or hemifused vesicles and by the roughness of the metal support. Thus, measurements of fluorescence recovery after photobleaching (FRAP) exclude the lateral mobility of a lipid monolayer on top of a thiolipid monolayer tethered to gold. ${ }^{4,8}$ Only small ionophores can be accommodated in the lipid bilayer moiety of gold-supported tBLMs, via incorporation from their aqueous solutions. ${ }^{9}$

It should also be noted that vesicles have a low propensity to fuse on the hydrophobic surface exposed to the aqueous solution by a gold-supported thiolipid monolayer, especially if they incorporate an integral protein; ${ }^{10,11}$ rather, they are adsorbed or partially fused. ${ }^{12,13}$ In this connection, incorporation of proton pumps in a gold-supported tBLM from their solutions in detergent may easily take place in the membrane of adsorbed or partially fused vesicles, since the vesicular membrane is clearly interposed between two aqueous phases. An analogous situation may be envisaged with proteoliposomes adsorbed or partially fused on a gold-supported thiolipid monolayer from their suspending solution. In this respect, the functional activity of the proton pumps investigated by Naumann et al. ${ }^{3-5}$ may well be successfully verified thanks to vesicles or proteoliposomes adsorbed and/or partially fused on the gold-supported thiolipid monolayer, even if the protein cannot span the lipid bilayer moiety of the tBLM. In fact, its activation may cause an increase in the proton concentration on top of the thiolipid monolayer (in the case of $\mathrm{F}_{0} \mathrm{~F}_{1}$ ATPase activated by ATP) or its decrease (in the case of COX activated by ferrocytochrome $c$ ). In view of the relative permeability of the leaky oligopeptide-based
tBLMs to protons, this may determine an increase or a decrease in the proton electroreduction current on gold, as actually observed. ${ }^{3,5}$

As distinct from gold or silver supports, mercury, thanks to its liquid state, provides a defect-free surface to the selfassembling film and imparts a high fluidity to the tBLM, allowing lateral mobility of the thiolipid molecules anchored to its surface. Thus, when the distal lipid monolayer of a mercury-supported DPTL|lipid tBLM or the lipid monolayer directly self-assembled on bare mercury consists of a raft-forming mixture, lipid microdomains are formed spontaneously and rapidly, as verified by differential capacitance measurements and by two-photon fluorescence lifetime imaging microscopy. ${ }^{14}$ In view of these advantageous features, DPTL/lipid tBLMs tethered to a hanging mercury drop electrode (HMDE) have been used to incorporate the ion carrier valinomycin, ${ }^{15}$ the channel-forming peptides melittin ${ }^{16}$ and gramicidin ${ }^{17}$ and the channel-forming proteins sarcolipin, ${ }^{18,19} \mathrm{OmpF}$ porin from Escherichia coli $^{20}$ and HERG potassium channel. ${ }^{21}$ The selfassembly of a lipid monolayer on top of the DPTL-coated mercury was carried out by immersing the mercury drop into an aqueous electrolyte on whose surface a lipid film had been previously spread, thus avoiding the use of vesicles.

Gold-supported DPTL|DPhyPC tBLMs on microchips (where DPhyPC stands for diphytanoylphosphatidylcholine) have been recently employed to record single-channel currents of peptides and proteins. To this end, a microelectrode array device consisting of many $\left(100 \times 100 \mu \mathrm{m}^{2}\right)$ "sensor" pads was employed. A DPTL monolayer was tethered to the gold-coated sensor pads from a DPTL solution in ethanol; a lipid monolayer was then formed on top of it by vesicle fusion. This device has allowed the recording of single-channel currents of gramicidin, ${ }^{22}$ the high-conducting $\mathrm{Ca}^{2+}$-activated $\mathrm{K}^{+}$(BK or Maxi-K) channel, the synthetic $\mathrm{M} 2 \delta$ ion channel $^{23}$ and the mechanosensitive channel of large conductance (MscL) from Escherichia coli. ${ }^{24}$ All these peptides and proteins were incorporated into lipid vesicles, before fusing them onto the DPTL-coated sensor pad. 


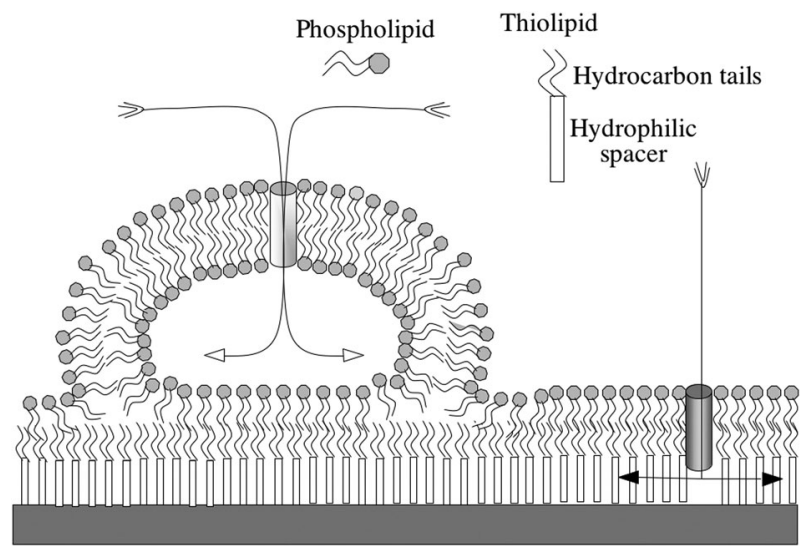

Fig. 2 Schematic picture of a tBLM with a partially fused vesicle on top; two alternative pathways of ionic flux are indicated, one into the hydrophilic spacer and the other into the partially fused vesicle. For convenience, the size of the partially fused vesicle has been reduced to about $1 / 3$ of its actual value, when compared with the tBLM thickness.

With the exclusion of the gramicidin channel, the unitary conductance of these ion channels was found to be from one-third to one-tenth of that obtained with conventional bilayer lipid membranes (BLMs). The channel lifetimes for both open and closed states were normally of the order of seconds and in good agreement with those obtained in BLMs under similar conditions.

An entirely analogous DPTL $\mid$ DPhyPC bilayer (tBL $\mu \mathrm{M}$ : tethered bilayer lipid micromembrane) was self-assembled by us on a mercury cap electrodeposited on a platinum microdisk, about $20 \mu \mathrm{m}$ in diameter. ${ }^{25}$ The distal DPhyPC monolayer was formed on the DPTL-coated mercury microcap by spilling a few drops of a DPhyPC solution in chloroform on it and by allowing the solvent to evaporate. The single-channel currents due to the opening of the channel protein OmpF porin from Escherichia coli were found to be quite different from those at a gold-supported DPTL|DPhyPC bilayer. Thus, they last only one or two milliseconds. Incidentally, the use of lipid-coated mercury microelectrodes electrodeposited on $\mathrm{Pt}$ was first introduced by Nelson and coworkers, ${ }^{26}$ who fabricated a wafer-based $\mathrm{Pt} \mid \mathrm{Hg}$ microarray coated with a phospholipid monolayer, for drug screening.

The present work aims at showing that single-channel currents with lifetimes analogous to those obtained at conventional BLMs, albeit smaller, are only obtained when ion channels are incorporated in lipid vesicles adsorbed or semi-fused on thiolipid monolayers self-assembled on microelectrodes. Fig. 2 shows a schematic picture of a tBLM with a partially fused vesicle on top; two alternative pathways of ionic flux are indicated, one into the hydrophilic spacer and the other into the partially fused vesicle.

\section{Experimental}

\subsection{Materials}

The water used was obtained from water produced by an inverted osmosis unit, upon distilling it once and then distilling the water so obtained from alkaline permanganate.
Merck (Darmstadt, Germany) suprapur ${ }^{(\mathbb{R}} \mathrm{KCl}$ was baked at $500{ }^{\circ} \mathrm{C}$ before use to remove any organic impurities. Diphytanoylphosphatidylcholine (DPhyPC), diphytanoylphosphatidylethanolamine (DPhyPE), dioleoylphosphatidylcholine (DOPC) and palmitoylsphingomyelin (PSM) were purchased in chloroform solution from Avanti Polar Lipids (Birmingham, AL). The 2,3,di-O-phytanyl-sn-glycerol-1-tetraethylene-glycol-D,L- $\alpha$ lipoic acid ester lipid (DPTL) was provided by Prof. Adrian Schwan (Department of Chemistry, University of Guelph, Canada). Alamethicin and cholesterol (Chol) were purchased from Sigma-Aldrich (Milano, Italy) and were used without further purification. Solutions of $0.2 \mathrm{mg} \mathrm{mL}^{-1}$ DPTL in ethanol were prepared from a $2 \mathrm{mg} \mathrm{mL}^{-1}$ solution of DPTL in ethanol. Stock solutions of this thiolipid were stored at $-18{ }^{\circ} \mathrm{C}$.

Small unilamellar vesicles (SUVs) were prepared by drying a single lipid or a lipid mixture in chloroform onto the walls of a test tube under flowing nitrogen for several hours, to ensure the removal of all the solvent. The dried lipid was resuspended in aqueous $0.1 \mathrm{M} \mathrm{KCl}$ by vortexing under a nitrogen atmosphere for about $20 \mathrm{~min}$. Lipid vesicles were formed by sonicating the suspension to clarity, using the $3 \mathrm{~mm}$ diameter microtip of an Autotune Series High Intensity Ultrasonic Processor (Sonics, Danbury, CT). To prevent heating the sample, the sonication was carried out on ice in 5 -s pulses separated by 5 -s cooling periods. The total sonication time was typically $45 \mathrm{~min}$. The SUVs were stored under nitrogen in small vials and were used over a period of one week. The hydrodynamic diameter of the vesicles measured by photon correlation spectroscopy (PCS) was $50 \mathrm{~nm}$, with a narrow distribution width.

\subsection{Preparation of the tethered bilayer lipid micromembrane (tBL $\mu \mathrm{M})$}

The tBL $\mu \mathrm{M}$ was obtained from a platinum wire, about $20 \mu \mathrm{m}$ in diameter, which was sealed in a glass capillary, $1 \mathrm{~mm}$ in outer diameter, with a P-2000 laser pipette puller (Sutter Instruments, S. Francisco, CA) and terminated with a platinum microdisk. The microdisk was polished with $0.3 \mu \mathrm{m}$ alumina on a 40-7218 Buehler polishing cloth. Mercury was then electrodeposited on the microdisk from a deaerated aqueous solution containing $10 \mathrm{mM} \mathrm{Hg}_{2}\left(\mathrm{NO}_{3}\right)_{2}, 0.1 \mathrm{M} \mathrm{KNO}_{3}$, and $1 \%$ (v/v) $\mathrm{HNO}_{3}$, to form a mercury spherical cap. The electrodeposition was monitored by recording the resulting faradaic charge against time; it was interrupted when the charge attained the value calculated for a height of the spherical cap equal to one half of the radius of its base. The spherical cap exhibited the same features as a normal liquid mercury electrode. The $\mathrm{Hg}$-coated $\mathrm{Pt}$ microdisk was immersed in a $0.2 \mathrm{mg} \mathrm{mL}^{-1}$ DPTL solution in ethanol for about $20 \mathrm{~min}$, to anchor a DPTL monolayer to the Hg surface. To form a lipid monolayer on top of the DPTL monolayer, a few drops of a lipid solution in chloroform were then spilled on the DPTL-coated mercury cap, allowing the solvent to evaporate. The resulting tBL $\mu \mathrm{M}$ was stabilized in aqueous $0.1 \mathrm{M} \mathrm{KCl}$ by continuous cyclic voltammetric scans between -0.200 and $-1.000 \mathrm{~V}$ vs. $\mathrm{Ag} \mid \mathrm{AgCl}$. Henceforth, this method of formation of the $\mathrm{tBL} \mu \mathrm{M}$ will be briefly referred to as the drop spilling method. Alternatively, the distal lipid monolayer was obtained 
by adding to a $0.1 \mathrm{M} \mathrm{KCl}$ aqueous solution containing a DPTL-coated mercury microcap an aliquot of vesicle preparation dosed to attain a vesicle concentration of $25 \mu \mathrm{g} \mathrm{mL}^{-1}$. The resulting $\mathrm{tBL} \mu \mathrm{M}$ was stabilized as previously described. The resistance $R$ and differential capacitance $C$ of the tBL $\mu \mathrm{M}$ were determined at $-0.5 \mathrm{~V}$ vs. $\mathrm{Ag} \mid \mathrm{AgCl}$ from its electrochemical impedance spectrum upon fitting it to an equivalent circuit consisting of a simple RC mesh. The resistance ranged from 1 to $10 \mathrm{G} \Omega$ and was, therefore, sufficiently high to allow the recording of single-channel activity by the patch-clamp technique. Its differential capacitance $C$ amounted to $0.8-1.0 \mu \mathrm{F} \mathrm{cm}{ }^{-2}$, which is a typical value for a well-behaved solvent-free lipid bilayer. Formation of lipid multilayers was excluded by verifying by electrochemical impedance spectroscopy that the conductivity of the $\mathrm{tBL} \mu \mathrm{M}$ in $0.1 \mathrm{M} \mathrm{KCl}$ is notably increased upon incorporating gramicidin. In fact, it is known that gramicidin acts as a channel toward monovalent inorganic cations in a lipid bilayer by forming a dimer; this is impossible in a lipid multilayer. It is quite probable that the lipid in excess accumulates on the rim of the mercury microcap, similarly to the formation of the Plateau-Gibbs border in black lipid membranes. After its preparation, the resistance and capacitance of the $\mathrm{tBL} \mu \mathrm{M}$ remained unaltered for at least one day. Even though the resistance of $1 \mathrm{G} \Omega$ for a $\mathrm{tBL} \mu \mathrm{M}$ is high enough for patch-clamp measurements, the corresponding specific resistance, about $5 \mathrm{k} \Omega \mathrm{cm}^{2}$, is much less than that attained with an identical tBLM anchored to a HMDE. In fact, the specific resistance of the latter tBLM, about $2 \times 10^{-2} \mathrm{~cm}^{2}$ in area, ranges from 5 to $10 \mathrm{M} \Omega \mathrm{cm}^{2}$. This apparent discrepancy is consistent with measurements of capacitance and resistance of DPTL $\mid$ DPhyPC tBLMs on gold microelectrodes of circular shape, with diameters ranging from $4 \times 10^{3}$ to $8 \mu \mathrm{m} .{ }^{27}$ For the larger electrodes, the capacitance is directly proportional to the electrode area, while the resistance is inversely proportional to it. For the smaller electrodes, the capacitance decreases linearly and the resistance increases linearly with a decrease in the electrode diameter. This indicates that capacitance and resistance of the larger electrodes are dominated by the electrode area, while the circumference seems to have the dominant role at smaller electrodes. Consequently, a slight disorder of the bilayer structure at the edge of a microelectrode has a major influence on its electrical properties.

Measurements at a tBLM anchored to a mercury electrode of large area $\left(2.1 \times 10^{-2} \mathrm{~cm}^{2}\right)$ were carried out with a homemade HMDE, described elsewhere. ${ }^{28}$ A home-made glass capillary with a finely tapered tip, about $1 \mathrm{~mm}$ in outer diameter, was employed. Capillary and mercury reservoir were thermostated at $25 \pm 0.1{ }^{\circ} \mathrm{C}$ in a water-jacketed box to avoid any changes in the drop area due to a change in temperature. Monolayers of DPTL were self-assembled on the HMDE by keeping the mercury drop immersed in a $0.2 \mathrm{mg} \mathrm{mL}^{-1}$ DPTL solution in ethanol for $20 \mathrm{~min}$. A monolayer consisting of a mixture of DPhyPC and DPhyPE in a 7:3 molar ratio was then formed on top of the DPTL monolayer by two different procedures. One procedure consisted in spreading a lipid solution in pentane on the surface of a $0.1 \mathrm{M} \mathrm{KCl}$ aqueous solution, in an amount corresponding to five to six phospholipid monolayers, and in allowing the pentane to evaporate. The DPTL-coated mercury drop was then immersed into the aqueous solution across the lipid film; this procedure causes a lipid monolayer to self-assemble on top of the DPTL monolayer, thanks to the hydrophobic interactions between the alkyl chains of the phospholipid and those of the thiolipid. The other procedure consisted in adding an aliquot of vesicle preparation, dosed to attain a vesicle concentration of $25 \mu \mathrm{g} \mathrm{mL}^{-1}$, to a $0.1 \mathrm{M} \mathrm{KCl}$ aqueous solution containing a DPTL-coated mercury drop and in allowing the vesicles to fuse on the DPTL monolayer. With both the "spreading procedure" and the "vesicle fusion procedure", the tBLM was then subjected to repeated potential scans over a potential range from -0.200 to $-1.200 \mathrm{~V}$ vs. $\mathrm{Ag} \mid \mathrm{AgCl}$ while continuously monitoring the curve of the quadrature component, $Y^{\prime \prime}$, of the electrode admittance at $75 \mathrm{~Hz}$ against the applied potential, $E$, using AC voltammetry, until a stable $Y^{\prime \prime}$ versus $E$ curve was attained.

\subsection{Instrumentation and patch-clamp measurements}

Impedance spectroscopy measurements were carried out with an Autolab instrument PGSTAT12 (Echo Chemie, Utrecht, The Netherlands) supplied with a FRA2 module for impedance measurements, SCAN-GEN scan generator and GPES 4.9007 Beta software. Potentials were measured vs. a $\mathrm{Ag} \mid \mathrm{AgCl}$ electrode immersed in a 0.1 or $0.5 \mathrm{M} \mathrm{KCl}$ working solution, but henceforth they will be referred to a saturated calomel electrode (SCE). Single-channel activity was recorded with an Axopatch 200B amplifier (Axon Instruments, Molecular Devices Corporation, Union City, CA). The $\mathrm{tBL} \mu \mathrm{M}$ and a silver wire coated with $\mathrm{AgCl}$ were immersed in a Petri dish containing an undeaerated $0.5 \mathrm{M} \mathrm{KCl}$ solution and both electrodes were connected to the amplifier. The signal was passed through a low-pass 0.1 or $0.5 \mathrm{kHz}$ filter and digitalized at a sampling frequency of 10 or $100 \mathrm{kHz}$ using a Digidata $1322 \mathrm{~A}$ (Molecular Devices Corporation, Union City, CA). The resistance of the $\mathrm{tBL} \mu \mathrm{M}$ was checked by carrying out a series of potential steps from a fixed initial potential of $-0.2 \mathrm{~V}$ to progressively more negative values, by $100 \mathrm{mV}$ increments. From the amplitude of the potential steps and from the resulting currents, the resistance was estimated using Ohm's law. After verifying that the resistance was $\geq 1 \mathrm{G} \Omega$, a few microlitres of $5 \times 10^{-4} \mathrm{M}$ alamethicin in water were added to the solution. After a waiting time of $10 \mathrm{~min}$ to permit the incorporation of the peptide in the lipid bilayer, the same series of potential steps was repeated.

\section{Results and discussion}

Alamethicin forms ion-conducting aggregates when the transmembrane potential is made sufficiently negative on the trans side of the membrane. In conventional BLMs, alamethicin conductance increases in discrete steps that form a pattern of five levels. ${ }^{29}$ The different conductance levels are ascribed to a different number of monomeric units forming the channel. ${ }^{30}$ Fluctuations in the channel size are considered to occur by uptake or release of single monomers by the channels. The open state of the alamethicin channels in BLMs may last a few seconds. In aqueous $0.1 \mathrm{M} \mathrm{KCl}$, the conductance due to the incorporation of $1 \mu \mathrm{M}$ alamethicin in BLMs undergoes an 


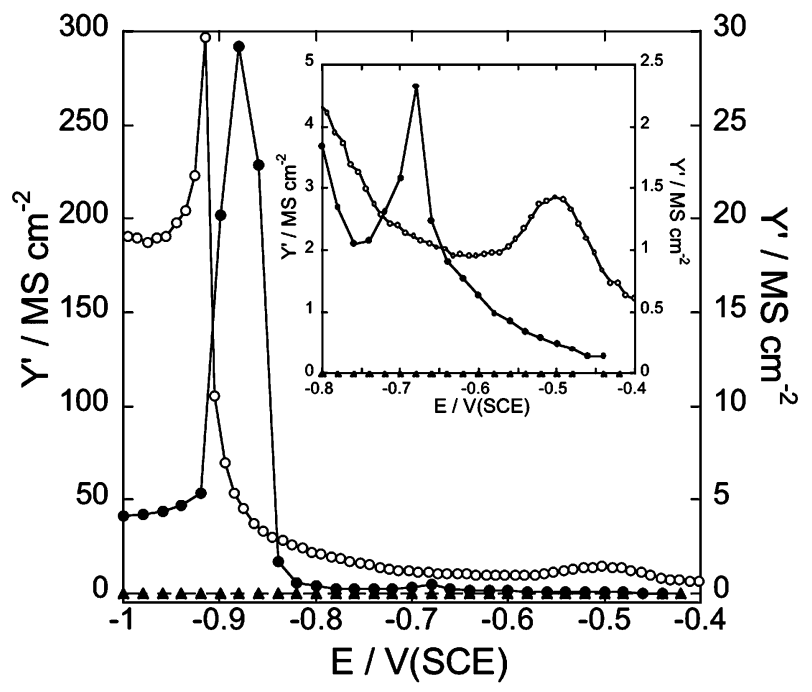

Fig. 3 Plot of the in-phase component, $Y^{\prime}$, of the admittance against the applied potential $E$ at $10 \mathrm{~Hz}$ for a mercury-supported DPTL $\mid(70$ mol\% DPhyPC + 30 mol\% DPhyPE) bilayer formed by the spreading procedure $(\bigcirc)$ or by vesicle fusion $(-)$ and immersed in a $0.1 \mathrm{M} \mathrm{KCl}$ aqueous solution containing $5 \times 10^{-6} \mathrm{M}$ alamethicin. The left-hand scale refers to $(\bigcirc)$, the right-hand one to $(\bullet)$. The inset shows an enlargement of the more positive section of the $Y^{\prime} v s . E$ plot. The $Y^{\prime}$ vs. $E$ plot in the absence of alamethicin is denoted by solid triangles and is referred to the left-hand scale; in this scale the plots obtained by the spreading procedure and by vesicle fusion are indistinguishable.

abrupt increase when the transmembrane potential attains a value of about $80 \mathrm{mV} .^{29}$

Preliminary conductance measurements were carried out on tBLMs supported by a HMDE. Electrochemical impedance spectra of Hg-supported DPTL|(70 mol\% DPhyPC + $30 \mathrm{~mol} \%$ DPhyPE) bilayers, formed by either the spreading or the vesicle-fusion procedure, were recorded in aqueous $0.1 \mathrm{M}$ $\mathrm{KCl}$ over a potential range from -0.3 to $-1.00 \mathrm{~V}$ and over a frequency range from 0.1 to $1 \times 10^{5} \mathrm{~Hz}$. The presence of alamethicin increases both the in-phase and the quadrature components of the tBLM admittance. Fig. 3 shows plots of the in-phase admittance, $Y^{\prime}$, against the applied potential $E$ at a constant frequency of $10 \mathrm{~Hz}$ for tBLMs obtained by both procedures, after incorporation of alamethicin from its $\mathrm{q} 5 \times 10^{-6} \mathrm{M}$ solution in aqueous $0.1 \mathrm{M} \mathrm{KCl}$. The $Y^{\prime}$ quantity at constant frequency is exactly proportional to the tBLM conductance only in the ideal situation in which the tBLM behaves like a pure combination of a resistance and a capacitance in parallel, i.e. a RC mesh. Since an accurate simulation of a tBLM requires an equivalent circuit with additional circuit elements, including the solution resistance, a plot of $Y^{\prime}$ against $E$ only provides a qualitative indication of the potential at which the tBLM undergoes a rapid increase in conductance (and also in capacitance) induced by an ion channel. $Y^{\prime}$ increases abruptly attaining a peak in the proximity of $-0.9 \mathrm{~V}$. The peak obtained with the tBLM prepared by the spreading procedure is slightly more negative and much higher than that obtained with the tBLM prepared by fusing vesicles. This is due to the fact that, in the latter case, alamethicin was added to an aqueous solution containing the vesicles, which

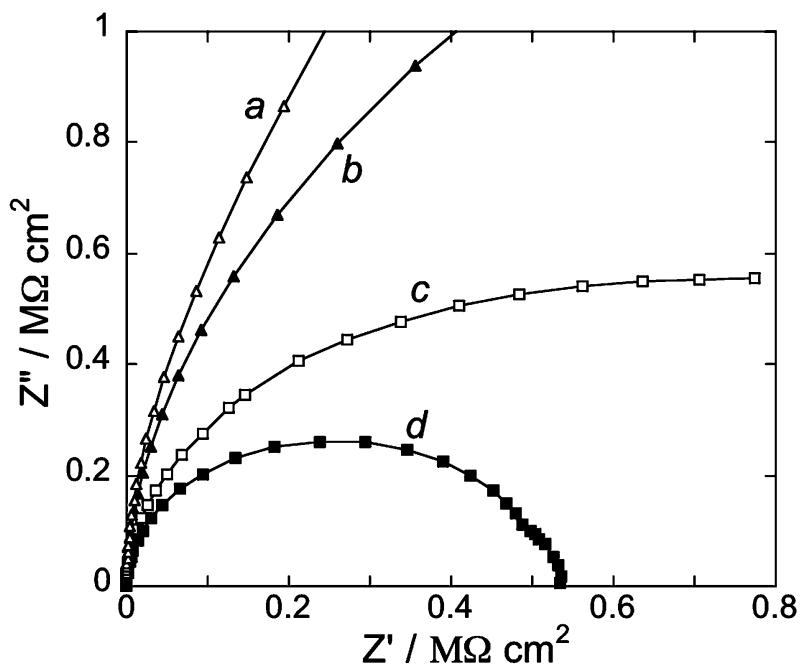

Fig. $4 Z^{\prime \prime}$ vs. $Z^{\prime}$ plot (Nyquist plot) for a mercury-supported DPTL $\mid(70$ mol $\%$ DPhyPC +30 mol\% DPhyPE) bilayer formed by the spreading procedure and immersed in a $0.1 \mathrm{M} \mathrm{KCl}$ aqueous solution, both in the absence ( $a$ at $-0.500 \mathrm{~V} ; c$ at $-0.800 \mathrm{~V}$ ) and in the presence of $5 \times 10^{-6} \mathrm{M}$ alamethicin $(b$ at $-0.500 \mathrm{~V} ; d$ at $-0.800 \mathrm{~V})$.

sequestered a large amount of the alamethicin molecules. Interestingly, $Y^{\prime}$ shows a slight increase at about $-0.7 \mathrm{~V}$ for the $\mathrm{tBLM}$ prepared by fusing vesicles and at about $-0.5 \mathrm{~V}$ for that prepared by the spreading procedure (see the inset of Fig. 3). Fig. 4 shows the Nyquist plot of a tBLM in $0.1 \mathrm{M} \mathrm{KCl}$ at $-0.500 \mathrm{~V}$ and $-0.800 \mathrm{~V}$, both in the absence and in the presence of $5 \times 10^{-6} \mathrm{M}$ alamethicin. These impedance spectra were fitted by a RC mesh, simulating the tBLM, with the resistance, $R_{\Omega}$, of the solution in series with it. In practice, the diameters of the roughly circular arcs in Fig. 4 measure the resistance $R$ of the tBLM. In the absence of alamethicin, $R$ decreases from 4.6 to $1.04 \mathrm{M} \Omega \mathrm{cm}^{2}$ in passing from -0.500 to $-0.800 \mathrm{~V}$. The presence of alamethicin decreases $R$ from 4.6 to $3.1 \mathrm{M} \Omega \mathrm{cm}^{2}$ at $-0.500 \mathrm{~V}$ and from 1.04 to $0.5 \mathrm{M} \Omega \mathrm{cm}^{2}$ at $-0.800 \mathrm{~V}$. In all four cases, the capacitance $C$ of the tBLM resulting from the fitting amounts to $0.65 \mu \mathrm{F} \mathrm{cm} \mathrm{cm}^{-2}$.

\subsection{Patch-clamp measurements of single-channel activity of alamethicin incorporated in $\mathrm{tBL} \mu \mathrm{Ms}$}

Incorporation of alamethicin in $\mathrm{tBL} \mu \mathrm{Ms}$ prepared by the drop spilling method yields single channel openings entirely analogous to those already obtained by incorporating $\mathrm{OmpF}$ porin in a $\mathrm{tBL} \mu \mathrm{M}$ prepared by the same method. ${ }^{25}$ Fig. 5 shows the current recorded as a function of time upon stepping the applied potential from a fixed initial potential of $-0.200 \mathrm{~V}$ to a final potential of $-0.500 \mathrm{~V}$ on a DPTL $\mid(70 \mathrm{~mol} \%$ DPhyPC $+30 \mathrm{~mol} \%$ DPhyPE) tBL $\mu \mathrm{M}$ immersed in aqueous $0.5 \mathrm{M} \mathrm{KCl}$ containing $1 \times 10^{-7} \mathrm{M}$ alamethicin. The singlechannel openings at the $\mathrm{tBL} \mu \mathrm{M}$ differ from those at a conventional BLM since they last only one or two milliseconds. This is ascribed to the fast local saturation of the hydrophilic spacer in the immediate vicinity of the channel mouth, due to the small thickness of the hydrophilic spacer (about $2 \mathrm{~nm}$ ). The current spikes attain a maximum value of about $100 \mathrm{pA}$, apart from one that is about twice as high and is also enlarged in the figure. The negative current spikes are due to the flow of a net 


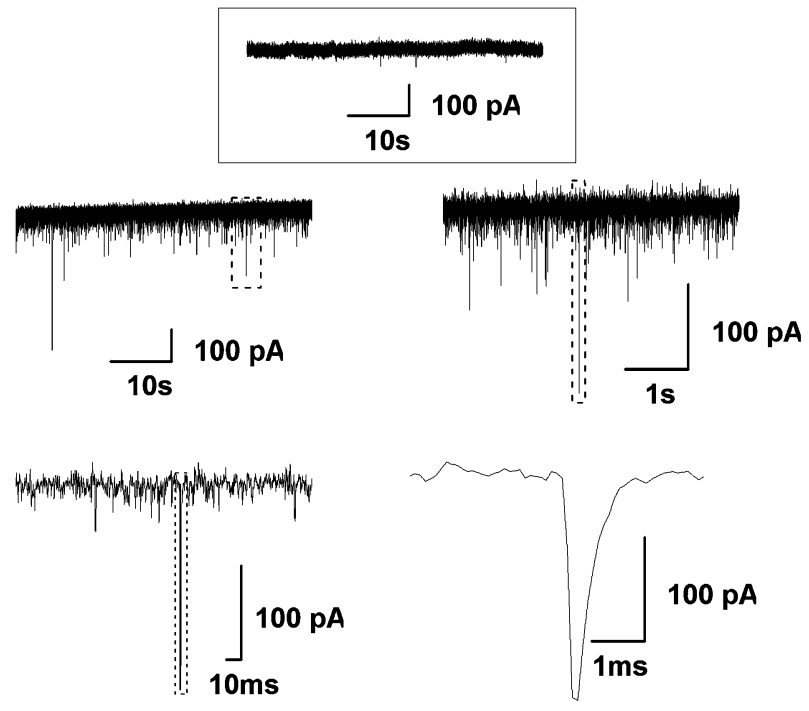

Fig. 5 Single channel activity from alamethicin channels incorporated in a DPTL $\mid(70 \mathrm{~mol} \%$ DPhyPC $+30 \mathrm{~mol} \%$ DPhyPE $)$ tBL $\mu \mathrm{M}$ formed by the drop spilling method and immersed in a $0.5 \mathrm{M} \mathrm{KCl}$ aqueous solution containing $1 \times 10^{-7} \mathrm{M}$ alamethicin, at an applied potential of $-0.700 \mathrm{~V} / \mathrm{SCE}$. Currents were recorded at a sampling frequency of $10 \mathrm{kHz}$ and filtered at $0.5 \mathrm{kHz}$. The first row refers to the $\mathrm{tBL} \mu \mathrm{M}$ in the absence of alamethicin. Proceeding from the second to the third row and from left to right, each trace is an enlargement of the section of the immediately preceding trace enclosed by a rectangle. The same is true for Fig. 6 and 7.

positive ionic charge into the hydrophilic TEO spacer. This flow of positive charge is compensated for by a flow of electrons to the electrode surface along the external circuit. It is the latter flow that is responsible for the current spikes. The electron charge is equal and opposite to the concomitant ionic charge, to maintain the overall electroneutrality of the mercury|solution electrified interface.

Several independent measurements carried out on DPTL $\mid$ DPhyPC bilayers supported by a HMDE have permitted us to relate the potential difference across the lipid bilayer moiety of the tBLM (i.e., the transmembrane potential $\phi_{2}$ ) to the applied potential measured $v s$. a SCE $;^{21,31,32}$ in the absence of ionic charges in the TEO moiety, $\phi_{2}$ is approximately given by $0.72 \times(E / \mathrm{SCE}+480 \mathrm{mV})$. Hence, alamethicin channel openings are mainly observed at potentials slightly negative of the zero transmembrane potential, with smaller openings at $-0.600 \mathrm{~V}$ and no current spikes at $-0.700 \mathrm{~V}$. Before incorporating alamethicin, no current spikes were observed by stepping the potential from $-0.2 \mathrm{~V}$ to final potentials ranging from -0.3 to $-0.9 \mathrm{~V}$. The single-channel openings are observed at potentials more positive than those at which the macroscopic conductance of the lipid bilayer moiety undergoes a sharp increase at a DPTL $\mid(70 \mathrm{~mol} \%$ DPhyPC + $30 \mathrm{~mol} \%$ DPhyPE) bilayer anchored to a HMDE. In fact, the macroscopic conductance in Fig. 3 starts to increase at about $-0.85 \mathrm{~V}$, which corresponds to a transmembrane potential of about $-0.250 \mathrm{~V}$. This behavior agrees with the conclusion by Latorre and Alvare ${ }^{33}$ that the alamethicin single-channel state distributions are only weakly voltage dependent: the macroscopic conductance induced by alamethicin mainly arises from an increase in the number of channels as the transmembrane potential becomes progressively more negative on the trans side of the membrane.

Incorporation of alamethicin in $\mathrm{tBL} \mu \mathrm{Ms}$ obtained by forming a lipid monolayer on top of a DPTL-coated mercury microcap by vesicle fusion yields single channel openings similar to those reported at conventional BLMs. However, the probability of their occurrence depends on the nature of the lipid used for the preparation of the vesicles. Thus, they occur only rarely using DPhyPC, but quite frequently using DOPC. The reason for the difference in behavior between DPhyPC and DOPC vesicles is probably to be ascribed to the fluidity that mercury imparts to the DPTL monolayer. This favors vesicle fusion, with sporadic formation of partially fused or adsorbed vesicles if there is no mismatch between the area occupied by the two phytanyl chains of the DPTL molecules and that occupied by the vesicular lipid molecules. In this respect, such a mismatch is expected to be smaller (or possibly absent) with DPhyPC vesicles than with vesicles prepared from phospholipids with unsaturated hydrocarbon chains. The role of this type of mismatch in disfavoring vesicle fusion was pointed out by Puu and Gustafson. ${ }^{12}$ Naturally, for the present purposes, we were interested in finding conditions favoring the formation of partially fused or adsorbed vesicles, rather than their fusion.

Fig. 6 and 7 show single channel activity from alamethicin incorporated in $\mathrm{tBL} \mu \mathrm{Ms}$ obtained by fusing DOPC vesicles on a DPTL-coated mercury microcap. The background electrical noise is higher than that observed on gold microelectrodes of comparable area, under otherwise identical conditions of electrode resistance; this is probably to be ascribed to the liquid mercury cap, which is subject to imperceptible vibrations. However, under favorable conditions, it was possible to achieve a background noise of about $4 \mathrm{pA}$ (see Fig. 7) by filtering at $0.1 \mathrm{kHz}$. At an alamethicin concentration of $1.8 \times 10^{-6} \mathrm{M}$, single channel currents of $\sim 60$ and $\sim 40 \mathrm{pA}$, with open probabilities of $\sim 40 \%$ and $\sim 10 \%$, respectively, were observed

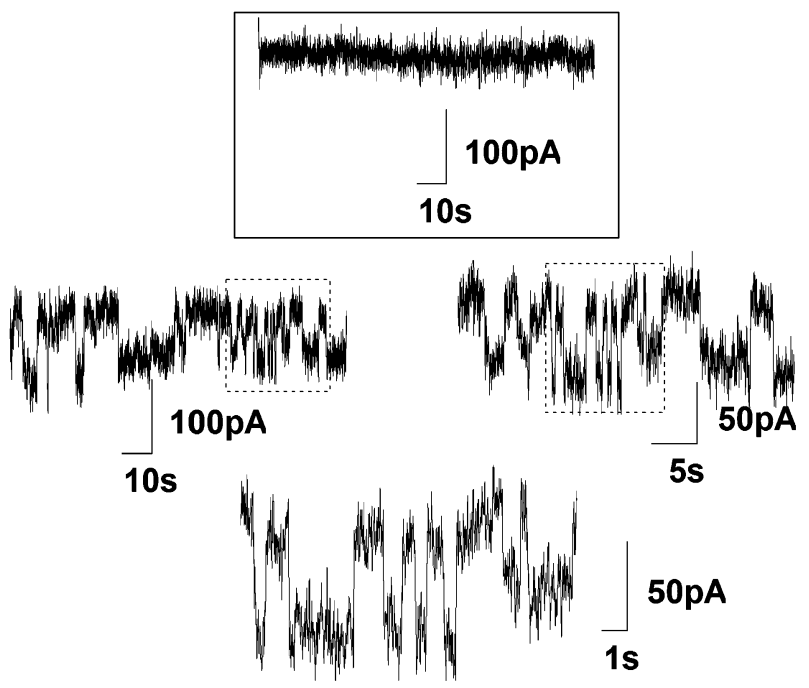

Fig. 6 Single channel activity from alamethicin channels incorporated in a DPTL $\mid \mathrm{DOPC} \mathrm{tBL} \mu \mathrm{M}$ formed by vesicle fusion and immersed in a $0.5 \mathrm{M} \mathrm{KCl}$ aqueous solution containing $1.8 \times 10^{-6} \mathrm{M}$ alamethicin, at an applied potential of $-0.500 \mathrm{~V} / \mathrm{SCE}$. Currents were recorded at a sampling frequency of $10 \mathrm{kHz}$ and filtered at $0.1 \mathrm{kHz}$. The first row refers to the $\mathrm{tBL} \mu \mathrm{M}$ in the absence of alamethicin. 


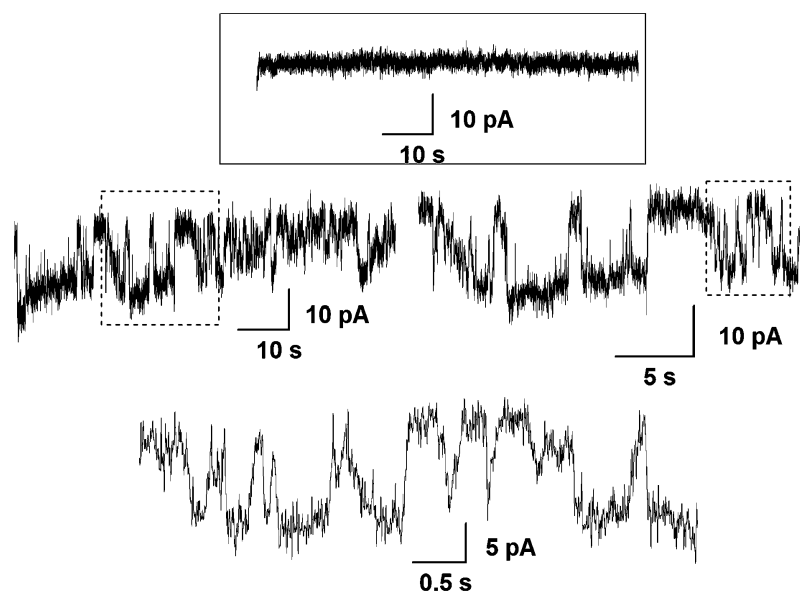

Fig. 7 Single channel activity from alamethicin channels incorporated in a DPTL $\mid$ DOPC $\mathrm{tBL} \mu \mathrm{M}$ formed by vesicle fusion and immersed in a $0.5 \mathrm{M} \mathrm{KCl}$ aqueous solution containing $2.5 \times 10^{-7} \mathrm{M}$ alamethicin, at an applied potential of $-0.500 \mathrm{~V} / \mathrm{SCE}$. Currents were recorded at a sampling frequency of $100 \mathrm{kHz}$ and filtered at $0.1 \mathrm{kHz}$. The first row refers to the tBLM in the absence of alamethicin.

(see Fig. 6). At a lower alamethicin concentration, single channel currents of $\sim 15 \mathrm{pA}$ were obtained (see Fig. 7). The most frequent open dwell times were in the 0.5-1 s range, but open dwell times as long as $20 \mathrm{~s}$ were also observed. However, we cannot exclude that the longer dwell times may include current spikes of duration shorter than the reciprocal of the cutoff frequency of the low-pass filter.

\subsection{Capacitive coupling between the vesicular membrane and the external circuit}

The single-channel currents recorded at DPTL|lipid bilayers supported by $\mathrm{Hg}$ or $\mathrm{Au}^{22-24}$ microelectrodes and obtained by vesicle fusion, albeit similar to those recorded at conventional BLMs, are characterized by a lower unitary conductance. This difference in behavior is readily explained if we assume that the single-channel currents are due to the opening of ion channels incorporated in the membrane of vesicles partially fused on the DPTL monolayer. Before the opening of an ion channel, the absolute potential difference $\Delta \phi$ across the whole interface, which differs from the applied potential $E$ by a constant, is given by:

$$
\Delta \phi=q_{\mathrm{M}}\left(C_{\text {DPTL }}^{-1}+C_{\mathrm{w}}^{-1}+C_{\mathrm{m}}^{-1}\right)
$$

Here $q_{\mathrm{M}}$ is the charge density on the metal surface; $C_{\mathrm{DPTL}}, C_{\mathrm{w}}$, $C_{\mathrm{m}}$ are the differential capacitances of the DPTL monolayer, the water within a partially fused vesicle and the vesicular membrane. As soon as the ion channel opens and translocates a charge $q$ (referred to the unit surface) across the vesicular membrane, the charge density on the metal varies from $q_{\mathrm{M}}$ to a different value $q_{\mathrm{M}}^{\prime}$, in order to maintain $\Delta \phi$ constant. Thus, $\Delta \phi$ is now given by:

$$
\Delta \phi=q_{\mathrm{M}}^{\prime}\left(C_{\mathrm{DPTL}}^{-1}+C_{\mathrm{w}}^{-1}\right)+\left(q_{\mathrm{M}}^{\prime}+q\right) C_{\mathrm{m}}^{-1}
$$

Combining eqn (1) and (2) we obtain the following expression for the charge that flows along the external circuit as a consequence of the opening of the ion channel:

$$
\begin{aligned}
\Delta q_{\mathrm{M}} & \equiv q_{\mathrm{M}}^{\prime}-q_{\mathrm{M}} \\
& =-q C_{\mathrm{DPTL}} C_{\mathrm{w}} /\left(C_{\mathrm{w}} C_{\mathrm{m}}+C_{\mathrm{DPTL}} C_{\mathrm{m}}+C_{\mathrm{DPTL}} C_{\mathrm{w}}\right)
\end{aligned}
$$

The charge $\Delta q_{\mathrm{M}}$ recorded by the patch-clamp amplifier is, therefore, less than that, $q$, flown across the vesicular membrane, and the same is true for the corresponding current.

Let us consider, as an example, the single-channel currents of the mechanosensitive MscL ion channel, recorded at a DPTL $\mid(70$ mol\% DPhyPC + 30 mol\% DPhyPE) bilayer tethered to a gold microelectrode, where lipid vesicles about $150 \mathrm{~nm}$ in diameter were employed. ${ }^{24}$ For a hemispherical partially fused vesicle with such a diameter, the capacitance $C_{\mathrm{w}}$ of the aqueous solution inside the vesicle, $75 \mathrm{~nm}$ in thickness, can be roughly estimated at $1.5 \mu \mathrm{F} \mathrm{cm}{ }^{-2}$. Giving $C_{\mathrm{tBLM}}$ and $C_{\mathrm{m}}$ the reasonable values 0.7 and $1 \mu \mathrm{F} \mathrm{cm}^{-2}$, respectively, the tBL $\mu \mathrm{M}$ should decrease the single-channel currents by a factor of 0.3 with respect to conventional BLMs. The estimated decrease would be greater in the case of vesicles simply adsorbed on the tBLM; in fact, in this case, an additional vesicular membrane is in direct contact with the DPTL monolayer. This decrease should be compared with the experimental decrease by about one order of magnitude, reported by Andersson et al. $^{24}$

\section{Conclusions}

Mercury-supported tBL $\mu \mathrm{Ms}$ were shown to yield single-channel recordings analogous to those obtained with conventional BLMs (albeit with a lower unitary conductance) if the distal lipid monolayer is formed by vesicle fusion, but not if it is formed by the drop-spilling method. This result indicates that these recordings are due to ionic flux into adsorbed and/or partially fused vesicles, and not into the hydrophilic spacer of the $\mathrm{tBL} \mu \mathrm{M}$. These conclusions can be readily extended to goldsupported $\mathrm{tBL} \mu \mathrm{Ms}$. Single-channel recordings due to ionic flux into the hydrophilic spacer are observed at mercury-supported tBL $\mu \mathrm{Ms}$ by using the drop-spilling method, in the absence of lipid vesicles; however, these recordings are different from those obtained by conventional BLMs, since they are quite sharp and last for one or two milliseconds. No such types of recording have ever been reported on gold-supported tBL $\mu \mathrm{Ms}$. This is due to the fact that the thiolipid molecules are rigidly bound to the gold surface atoms, thus preventing the incorporation of membrane proteins into gold-supported tBL $\mu \mathrm{Ms}$ and macroscopic tBLMs. Only small ionophores, such as valinomycin, can be incorporated in gold-supported tBLMs. ${ }^{9}$ Nonetheless, the functional activity of proton pumps immobilized on leaky gold-supported tBLMs formed by vesicle fusion can be successfully monitored, ${ }^{3-5}$ thanks to the presence of adsorbed and/or partially fused vesicles incorporating the proton pump. The situation is different with mercury-supported tBLMs, because the liquid nature of mercury allows the thiolipid molecules to move laterally, thus making room even for large membrane proteins such as the HERG potassium channel ${ }^{21}$ and the OmpF porin. ${ }^{20}$

In conclusion, both gold- and mercury-supported tBL $\mu \mathrm{Ms}$ with the distal lipid monolayer formed by vesicle fusion can monitor the functional activity of channel proteins and their voltage dependence, if any. They can also monitor the activity of drugs (inhibitors, agonists, antagonists) on channel proteins. An advantage over BLMs consists in their being robust, movable, easily prepared and, therefore, disposable. This may open the 
way to the realization of a tBL $\mu \mathrm{M}$ microarray platform for highly parallel screening of a large set of drugs and diagnostic targets on channel proteins.

\section{Acknowledgements}

The financial support by Ente Cassa di Risparmio di Firenze and that by the Italian Ministero dell'Istruzione, dell'Università e della Ricerca (MIUR) through PRIN 20079Y9578 are gratefully acknowledged. The authors are indebted to Prof. Elisabetta Cerbai (Department of Pharmacology, Florence University, Italy) for invaluable advise about the patch-clamp measurements and for the use of the Axopatch 200B amplifier.

\section{References}

1 R. Guidelli, G. Aloisi, L. Becucci, A. Dolfi, M. R. Moncelli and F. Tadini Buoninsegni, J. Electroanal. Chem., 2001, 504, 1-28, and references therein.

2 S. M. Schiller, R. Naumann, K. Lovejoy, H. Kunz and W. Knoll, Angew. Chem., Int. Ed., 2003, 42, 208-211.

3 R. Naumann, A. Jonczyk, C. Hampel, H. Ringsdorf, W. Knoll, N. Bunjes and P. Gräber, Bioelectrochem. Bioenerg., 1997, 42, 241-247.

4 R. Naumann, T. Baumgart, P. Gräber, A. Jonczyk, A. Offenhäusser and W. Knoll, Biosens. Bioelectron., 2002, 17, 25-34.

5 R. Naumann, E. K. Schmidt, A. Jonczyk, K. Fendler, B. Kadenbach, T. Liebermann, A. Offenhäusser and W. Knoll, Biosens. Bioelectron., 1999, 14, 651-662.

6 I. K. Vockenroth, C. Ohm, J. W. F. Robertson, D. J. McGillivray, M. Lösche and I. Köper, Biointerphases, 2008, 3, FA68-FA73.

7 J. Leitch, J. Kunze, J. D. Goddard, A. L. Schwan, R. J. Faragher, R. Naumann, W. Knoll, J. R. Dutcher and J. Lipkowski, Langmuir, 2009, 25, 10354-10363.

8 T. Baumgart, M. Kreiter, H. Lauer, R. Naumann, G. Jung, A. Jonczyk, A. Offenhäusser and W. Knoll, J. Colloid Interface Sci., 2003, 258, 298-309.

9 R. Naumann, D. Walz, S. M. Schiller and W. Knoll, J. Electroanal. Chem., 2003, 550-551, 241-252.

10 A. Zebrowska and P. Krysinski, Langmuir, 2004, 20, 11127-11133.
11 L. J. C. Jeuken, S. D. Connell, P. J. F. Henderson, R. B. Gennis, S. D. Evans and R. J. Bushby, J. Am. Chem. Soc., 2006, 128, 1711-1716.

12 G. Puu and I. Gustafson, Biochim. Biophys. Acta, Biomembr., 1997, 1327, 149-161.

13 J. Jass, T. Tjärnhage and G. Puu, Biophys. J., 2000, 79, 3153-3163.

14 L. Becucci, S. Martinuzzi, E. Monetti, R. Mercatelli, F. Quercioli, D. Battistel and R. Guidelli, Soft Matter, 2010, 6, 2733-2741.

15 L. Becucci, M. R. Moncelli, R. Naumann and R. Guidelli, J. Am. Chem. Soc., 2005, 127, 13316-13323.

16 L. Becucci, R. Romero Léon, M. R. Moncelli, P. Rovero and R. Guidelli, Langmuir, 2006, 22, 6644-6650.

17 L. Becucci, A. Santucci and R. Guidelli, J. Phys. Chem. B, 2007, 111, 9814-9820.

18 L. Becucci, R. Guidelli, C. B. Karim, D. D. Thomas and G. Veglia, Biophys. J., 2007, 93, 2678-2687.

19 L. Becucci, R. Guidelli, C. B. Karim, D. D. Thomas and G. Veglia, Biophys. J., 2009, 97, 2693-2699.

20 L. Becucci, M. R. Moncelli and R. Guidelli, Langmuir, 2006, 22, 1341-1346.

21 L. Becucci, M. V. Carbone, T. Biagiotti, M. D’Amico, M. Olivotto and R. Guidelli, J. Phys. Chem. B, 2008, 112, 1315-1319.

22 M. Andersson, H. K. Keizer, C. Zhu, D. Fine, A. Dodabalapur and R. S. Duran, Langmuir, 2007, 23, 2924-2927.

23 H. M. Keizer, B. R. Dorvel, M. Andersson, D. Fine, R. B. Price, J. R. Long, A. Dodabalapur, I. Köper, W. Knoll, P. A. V. Anderson and R. S. Duran, ChemBioChem, 2007, 8, 1246-1250.

24 M. Andersson, G. Okeyo, D. Wilson, H. Keizer, P. Moe, P. Blount, D. Fine, A. Dodabalapur and R. S. Duran, Biosens. Bioelectron., 2008, 23, 919-923.

25 L. Becucci, M. D'Amico, S. Daniele, M. Olivotto, A. Pozzi and R. Guidelli, Bioelectrochemistry, 2010, 78, 176-180.

26 Z. Coldrick, P. Steenson, P. Millner, M. Davies and A. Nelson, Electrochim. Acta, 2009, 54, 4954-4962.

27 I. K. Vockenroth, D. Fine, A. Dodobalapur, A. T. A. Jenkins and I. Köper, Electrochem. Commun., 2008, 10, 323-328.

28 M. R. Moncelli and L. Becucci, J. Electroanal. Chem., 1997, 433, 91-96.

29 M. Eisenberg, J. E. Hall and C. A. Mead, J. Membr. Biol., 1973, 14, 143-176.

30 G. Boheim, J. Membr. Biol., 1974, 15, 277-303.

31 L. Becucci, M. R. Moncelli and R. Guidelli, Langmuir, 2003, 19, 3386-3392.

32 R. Guidelli and L. Becucci, Soft Matter, 2011, 7, 2195-2201.

33 R. Latorre and O. Alvarez, Physiol. Rev., 1981, 61, 77-150. 